\title{
Tracking the Brain's Functional Coupling Dynamics over Development
}

\author{
R. Matthew Hutchison ${ }^{1}$ and J. Bruce Morton ${ }^{2}$ \\ ${ }^{1}$ Center for Brain Science, Harvard University, Cambridge, Massachusetts 02138, and ${ }^{2}$ Department of Psychology, University of Western Ontario, London, \\ Ontario N6A 3K7, Canada
}

The transition from childhood to adulthood is marked by pronounced functional and structural brain transformations that impact cognition and behavior. Here, we use a functional imaging approach to reveal dynamic changes in coupling strength between networks and the expression of discrete brain configurations over human development during rest and a cognitive control task. Although the brain's repertoire of functional states was generally preserved across ages, state-specific temporal features, such as the frequency of expression and the amount of time spent in select states, varied by age in ways that were dependent on condition. Increasing age was associated with greater variability of connection strengths across time at rest, while there was a selective inversion of this effect in higher-order networks during implementation of cognitive control. The results suggest that development is characterized by the modification of dynamic coupling to both maximize and constrain functional variability in response to ongoing cognitive and behavioral requirements.

Key words: brain states; cognitive control; fMRI; neural noise; resting state

\section{Introduction}

The abilities to maintain task goals and to filter distracting information are aspects of cognitive control (CC) that undergo profound change in early development (Zelazo, 2004; Luna et al., 2010; Somerville and Casey, 2010; Diamond, 2013). In the adult brain, these processes are rooted in the emergent dynamics of highly interconnected-and interacting-networks (Bullmore and Sporns, 2009). The spatiotemporal properties of the brain that constrain inter-regional dynamics, including anatomical coupling length, transmission time delays, and neurophysiological noise (Deco et al., 2009), change dramatically in early development as a consequence of a myriad of structural and functional alterations (for review, see Andersen, 2003; Brenhouse and Andersen, 2011) that impact both cognition and behavior. Indeed, numerical simulation and empirical electrophysiological investigations have highlighted the critical importance of internal dynamics for optimized brain function, and have shown, among other things, that age-related increases in behavioral speed and

Received Nov. 10, 2014; revised March 21, 2015; accepted March 24, 2015.

Author contributions: R.M.H. and J.B.M. designed research; R.M.H. and J.B.M. performed research; R.M.H. and J.B.M. contributed unpublished reagents/analytic tools; R.M.H. and J.B.M. analyzed data; R.M.H. and J.B.M. wrote the paper.

This research was supported by grants from the National Science and Engineering Research Council, the Canadian Foundation for Innovation, and the Ontario Innovation Trust to J.B.M.; and a Canadian Institute of Health Research postdoctoral fellowship and a Brain \& Behavior Research Foundation NARSAD Young Investigator Grant to R.M.H. We thank Elena Allen for sharing code used in the analysis, and Katie Insel for comments on an earlier version of the manuscript.

The authors declare no competing financial interests.

Correspondence should be addressed to Dr. J. Bruce Morton, Cognitive Development and Neuroimaging Laboratory, University of Western Ontario, London, 0N N6A 3K7, Canada. E-mail: bmorton3@uwo.ca or jbrucemorton@gmail.com.

DOI:10.1523/JNEUROSCI.4638-14.2015

Copyright $\odot 2015$ the authors $\quad 0270-6474 / 15 / 356849-11 \$ 15.00 / 0$ stability are associated with higher levels of neurophysiological noise and more complex brain responses to external stimulation (McIntosh et al., 2008). Thus, age-related differences in wholebrain emergent dynamics and their possible association with cognitive control deserve closer consideration as they could serve as important markers of developmental trajectories (indexing system integrity and function; Di Martino et al., 2014).

Age-related differences in inter-regional interaction-or functional connectivity (FC) - have been examined at a macroscopic, whole-brain level using neuroimaging techniques, and are linked to cognitive and behavioral maturation. Several intrinsic connectivity networks (ICNs) widely recognized from the study of adults-including prefrontal, default, sensory-motor, and salience networks - are evident early in development. These ICNs undergo topological reorganization over time (Supekar et al., 2009; Power et al., 2010), including the strengthening of longrange connections and the weakening of short-range connections within cognitive control networks (Fair et al., 2007), strengthening of the temporal segregation (anti-correlation) of task-positive and default networks (Marsh et al., 2006; Thomason et al., 2008), and a shift in location of cortical hubs from primary sensorymotor to heteromodal cortex (Fransson et al., 2011). While important, this picture of development overlooks the dynamic nature of the brain's functional connectivity profile, owing in part to the assumption that FC profiles are spatiotemporally invariant during the measurement period. Analysis strategies more sensitive to inter-regional coupling dynamics reveal short-lived meta-stable FC states that are reproducible across subjects and that deviate substantially from canonical connectivity patterns (Liu et al., 2013; Allen et al., 2014). The goal of the present study then was to examine age-related differences in FC dynamics and their possible association with behavior. 
A Conditions

Rest

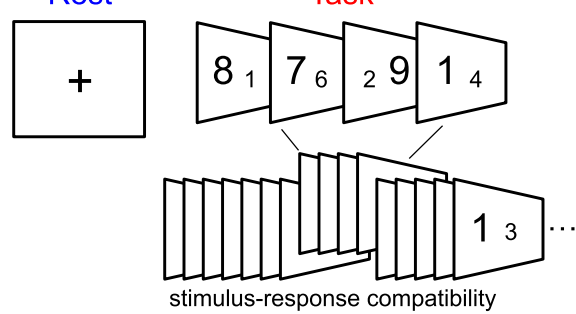

B ICN identification

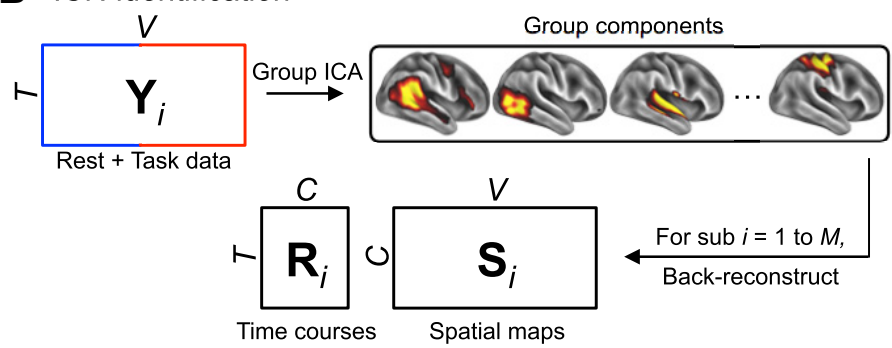

C Sliding window correlation

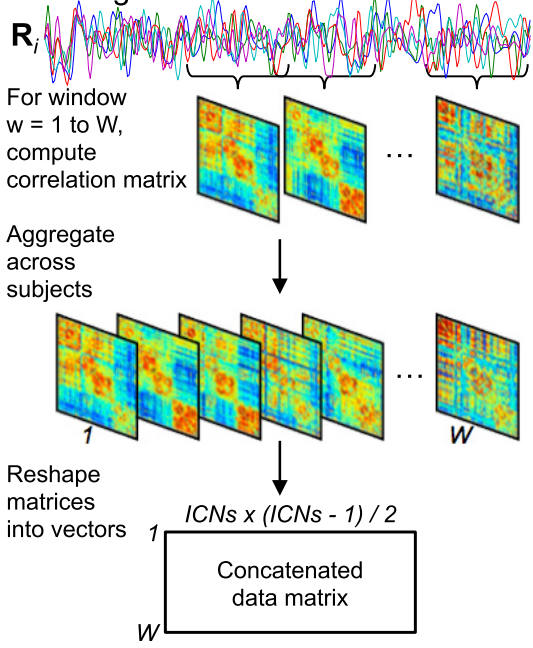

D State detection

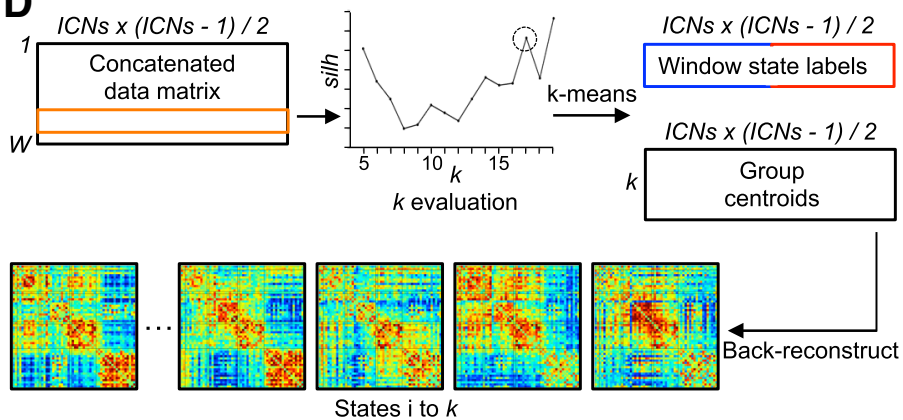

E Inter-ICN variability over time

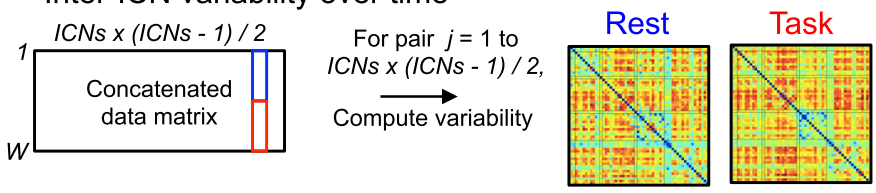

Figure 1. Illustration of task and analysis steps. $A$, Subjects were scanned in two different conditions, with eyes open at rest while fixating and during a size-congruency task administered in the form of a conflict adaptation paradigm. In the latter, on individual trials, participants were presented with two digits that differed in physical and numerical size, and pressed a button corresponding to the location of the numerically larger digit. On compatible trials, the numerically larger digit was also physically larger. On incompatible trials, the numerically larger digit was physically smaller. Individual trials were administered in conditions that differed in the proportion of overall trials that were compatible ( $25 \%$ or $75 \%)$. B, Data across subjects and conditions were decomposed using Group ICA into $C=90$ components, 56 of which were identified as ICNs. GICA1 back-reconstruction was used to estimate the time courses $\left(R_{i}\right)$ and spatial maps $\left(S_{i}\right)$ for each subject. $C$, Dynamic FC was estimated as the series of correlation matrices from windowed portions $(W)$ of $R_{i r}$ resulting in a concatenated data matrix of all ICN-to-ICN paired correlation values over time. $D$, Estimation of $k$ was performed to select an optimal cluster number for clusters and then, following steps outlined in Allen et al., 2014, $k$-means was performed on the concatenated data matrix, resulting in a labeling of each window to a cluster and cluster centroids (state) that can be back-projected into the original matrix format. $E$, Variance in the correlation strength of each IC-to-IC coupling pair (IC2ICVar) was computed separately for the two conditions.

We had two predictions. First, given that structural constraints on emergent brain dynamics change over development, we predicted age-related differences in the temporal expression of whole-brain connectivity states that comprise the functional repertoire. Second, given age-related advances in the ability to respond to unpredicted challenges and hypothesized links between flexible cognition and the complexity of evoked brain dynamics, we predicted that inter-regional connectivity dynamics that occur in association with efficient stimulus/response selection and stable task-set maintenance would be more complex among older compared with younger individuals.

\section{Materials and Methods}

All procedures were approved by the University Research Ethics Board for Health Sciences Research at the University of Western Ontario (London, ON, Canada) and are in accordance with the 1964 Declaration of Helsinki. Portions of the data used in this study have been published previously (Wilk and Morton, 2012; Wilk et al., 2012). An overview of the methodological procedure is illustrated in Figure 1.

Subjects. Fifty-one individuals ( 29 males) ranging in age from 9.4 to 32.4 years (mean, 16.9 years; SD, 5.8 years) participated, including 32 children ( $\leq 18$ years of age) and 19 adults ( $>18$ years of age). Participants $<18$ years of age were recruited from the University of Western Ontario Child Development Participant Pool. Parents of these participants gave written consent to their child's participation, and children provided verbal assent. Participants $\geq 18$ years of age were recruited from the University of Western Ontario undergraduate and graduate student populations, and provided written consent to their participation. All participants were right handed and had normal or corrected-tonormal vision.

Resting-state protocol. Participants were instructed to remain fixated on a centrally presented cross for 6 min and allow their thoughts to flow freely.

Task paradigm. In each of three 7.8 min runs, participants were administered 76 trials of a standard stimulus-response compatibility task (Henik and Tzelgov, 1982; Fig. 1A). On each trial, two Arabic digits differing in physical size and numerical magnitude were simultaneously presented for $1950 \mathrm{~ms}$ in white on a black background. Digits ranged in value from 1 to 9. Physically larger and smaller digits were presented in 60- and 30-point font, respectively. On compatible trials, the numerically larger digit was physically larger; on incompatible trials, the numerically larger digit was physically smaller. Participants selected, by means of a righthanded button press, the numerically larger stimulus. Individual trials were randomly jittered by means of an intertrial interval (ITI; range, $1500-4500 \mathrm{~ms}$; mean, $3000 \mathrm{~ms}$ ) and were ordered to ensure that $50 \%$ of trials were compatible. To improve the signal-to-noise ratio, $118000 \mathrm{~ms}$ null events were added to each run. A fixed random run, trial, ITI, and null event ordering was used for all participants. The paradigm was presented using E-Prime software (Psychology Software Tools).

Data acquisition. Data were collected using a 3 T Siemens Tim Trio MRI system fitted with a Siemens 32-channel head coil. Before scanning, 
participants $<18$ years of age received training in a mock-scanner facility to help limit motion and anxiety during the actual imaging procedure. Four functional runs were collected from each participant in a single scanning session, a single 180 -volume resting-state run (eyes open, fixation) and three 234-volume task runs. Functional volumes consisted of 32 slices (thickness, $3 \mathrm{~mm}$ ) oriented to the anterior commissure-posterior commissure axis and were collected in an ascending interleaved order using a T2*-weighted echoplanar imaging sequence (TR, $2000 \mathrm{~ms}$; $\mathrm{TE}, 30 \mathrm{~ms}$; flip angle, $78^{\circ}$ ), with an in-plane matrix of $64 \times 64$ pixels and a $21.1 \times 21.1 \mathrm{~cm}$ field of view, yielding a $3 \times 3 \times 3 \mathrm{~mm}$ voxel resolution. There were no gaps between slices. To assist in the visualization of functional analyses, a T1-weighted anatomical scan consisting of 192 slices (thickness, $1 \mathrm{~mm}$ ) with an in-plane $256 \times 256$ matrix and a $21.1 \times 21.1$ $\mathrm{cm}$ field of view (yielding a $1 \times 1 \times 1 \mathrm{~mm}$ voxel resolution) was also collected as part of the same scanning session.

Data preprocessing. fMRI volumes were corrected for motion, smoothed with an $8 \mathrm{~mm}$ Gaussian kernel, and normalized to the MNI template using SPM8.

Independent component analysis. Preprocessed volumes of concatenated resting-state and task data were submitted to high-model order group ICA using GIFT software (http://icatb.sourceforge.net; Calhoun et al., 2001; Fig. 1B). Two principal components analysis steps reduced the data to 90 components. Group data were then unmixed into 90 maximally independent components (ICs) using the Infomax algorithm. A high model order was used to improve the detection of noise sources and to limit the grouping of related subnetworks. Individual subject components were then created through back-reconstruction. Fifty-six neurophysiologically plausible sources, or ICNs, were identified by two trained viewers through independent visual (VIS) inspection of the spatial maps of the group ICs (Fig. 2). Plausible sources included auditory (AUD), somatomotor (SM), subcortical (SC), CC, cerebellar (CB), VIS, and default mode (DN) ICs. IC time courses were then filtered using a Butterworth filter and were despiked by replacing values greater than \pm 3 SDs with values equal to \pm 3 SDs. The group-averaged correlation matrix was calculated among all ICs following a Fisher $z$-transformation and the mean calculation of each subject's set of scans (Fig. 3).

Behavioral assessment. Task performance was measured in terms of response time (RT) variability and error rate separately for each participant. RT variability was based on all trials with correct responses and was computed as the average squared deviation of each individual response time from the mean of all response times. The error rate was computed as the total number of errors divided by the total number of trials.

Mean FC estimation. The mean FC was estimated by cross-correlating the full time courses of all 56 neurophysiologically plausible ICNs (Fig. 3). Age-related differences in the mean FC were estimated as the partial correlation of age and mean FC, with subject motion as a controlling variable (Fig. 4).

Dynamic matrix computation. Internetwork dynamic FC analysis was performed on the 56 filtered and despiked IC time courses by means of a sliding window technique (Fig. 1C). Using a window of $30 \mathrm{~s}$ width (15 TRs), the time points of all 56 IC time courses within the window were cross-correlated using Pearson's correlation coefficient, yielding a total of 1540 unique IC-to-IC correlations. For each participant, these correlations were computed a total of 750 times (150 times from rest data, 600 times from task data) by sliding the window along subject-specific time courses using a time step of $2 \mathrm{~s}$ (1 TR).

Dynamic state detection. Concatenated matrices across all 51 participants were submitted to a $k$-means cluster analysis (Fig. 1D). The $k$-means solution was evaluated across values of $k$ ranging from 5 to 20 using the silhouette metric. The $k$ value that returned the highest silhouette score without overfitting the data was 17 (Fig. 1D). Of the 17 total states, 5 were found to be unique to individual participants and were not considered, and the windows assigned to these states omitted from future analysis. Thus, our analysis focused on 12 connectivity states that dynamically reoccurred over time and were observable across multiple participants.

The following four metrics assessed connectivity state expression separately for each participant during rest and task: (1) frequency $(F)$, measured as the proportion of all windows classified as instances of particular states, and computed separately for each of the 12 states; (2) mean dwell time (MDT), measured as the average number of consecutive windows classified as instances of the same state, and computed separately for each of the 12 states; (3) intertransition interval, measured as the number of consecutive instances before a transition to a different state and computed as an average across all states; and (4) the number of transitions (NT), measured as the number of state transitions across rest and task conditions.

To test the association between age and connectivity state expression while controlling for interindividual differences in motion, we modeled frequency, mean dwell time, intertransition interval, and number of transitions each separately as weighted linear sums of age and motion averaged across rest and task. Motion was computed separately for each participant as the average root mean square of the three translation and three rotation estimates generated during motion correction. To examine the effect of condition (rest vs task) and the interaction of age and condition, connectivity state metrics were computed from rest and task data separately. The effect of condition was then tested by means of paired-sample $t$ tests. To test the interaction of age and condition, state expression metrics were modeled as weighted linear sums of age and motion separately for rest and task. We then tested whether the magnitude of the $\beta$-coefficient for age differed across rest and task conditions. To test whether connectivity state expression was further explainable by behavioral performance and sex, we added RT variability, error rate, and sex to the models, and tested whether these variables explained any additional variance in state expression variables. Scatterplots showing the association of age with the frequency and mean dwell time of each state separately for rest (blue circles) and task (red circles) are shown in Figure 5.

Assessment of internetwork correlation variability. For each pairwise ICN-to-ICN coupling, variability in coupling strength was estimated as the variance of the ICN-to-ICN correlation (Fig. 1E), and was computed separately for rest and task data. Associations among internetwork correlation variability, age, and behavioral performance were examined in the context of multiple regression models in which internetwork correlation variability was modeled as the weighted sum of age, condition (task vs rest), age $\times$ condition, $\mathrm{RT}$ variability, and error rate. To control for the possible contaminating effects of subject motion known to differ across age (Power et al., 2012; Satterthwaite et al., 2012; Van Dijk et al., 2012; Yan et al., 2013), subject-wise root mean square motion was included as a nuisance regressor in all models.

\section{Results}

\section{Behavior}

RT variability decreased with increasing age $\left(r_{(49)}=-0.35, p<\right.$ $0.05)$. The error rate across all trials was unrelated to age $\left(r_{(49)}=\right.$ -0.17 , not significant).

\section{Mean FC}

Analysis of the mean FC revealed a canonical pattern of interregional connectivity, including positive on-diagonal correlations among functionally related ICs; positive off-diagonal correlations between SM, CC, and VIS ICs; and negative offdiagonal correlations with DN ICs (Fig. 3). Static/mean IC-to-IC FC was associated with age (Fig. 4). The strongest associations were positive, and were evident largely among CC ICs, and between SM and VIS ICs. Weaker negative associations were particularly evident between CC and DN ICs.

\section{Connectivity states}

Twelve states dynamically reoccurred over time and were observable across multiple participants [Fig. 5 (for labels, see Fig. 3)]. Participants expressed an average of 4.15 (SD, 1.67) and 4.25 (SD, 1.96) states, respectively, during rest and task. The number of states expressed at rest was uncorrelated with age, but the number 


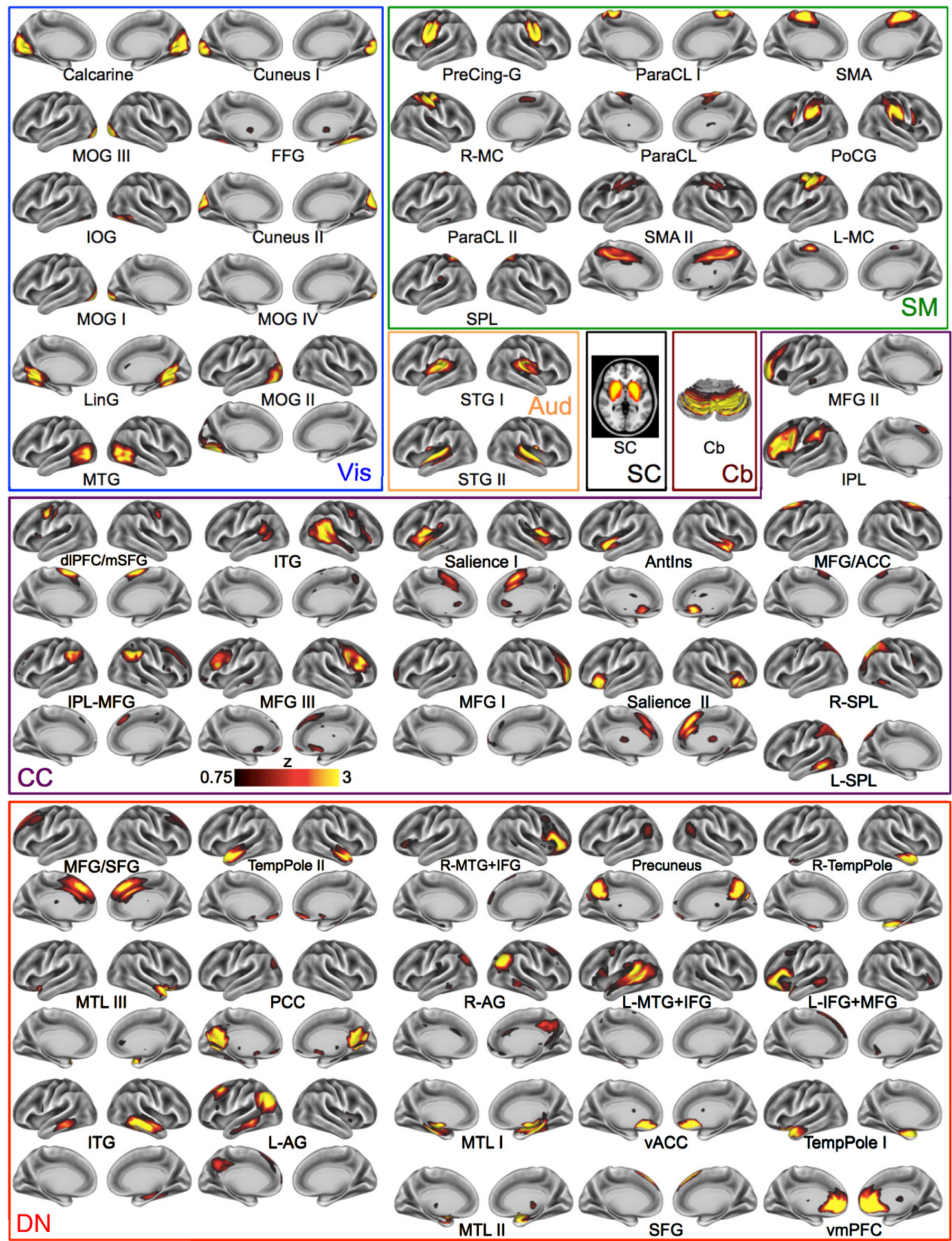

Figure 2. Spatial profiles of the 56 neurophysiologically plausible ICNs revealed by a high-model order group ICA displayed on the medial and lateral views of the cortical surface. ICNs are grouped, and boxes are color coded based on their assignment, which is shown in Figure 3 . In cases where a view or hemisphere is missing, no voxels above the lower-bound threshold $(z>0.75)$ were present.

of states expressed during a task was positively associated with age $\left(r_{(49)}=0.3, p<0.03\right)$.

Of the 12 states that were observable across participants, one state (state A) closely resembled the mean FC, with strong positive correlations along the diagonal (i.e., within functional groupings), and strong off-diagonal anti-correlations between CC and DN networks (Fig. 3). The remaining 11 states differed markedly from this pattern with differences between states both within and between functional groupings. Among SM networks, coupling ranged from being highly integrated (e.g., state C) to relatively disintegrated (e.g., state J), with some subclustering involving right and left motor cortex, superior parietal lobule, paracentral lobule, and postcentral gyrus evident in state E. Among CC networks, there was a departure away from one broadly integrated pattern evident in state A to two subclusters, one that included dorsolateral prefrontal cortex (dlPFC), ante- 


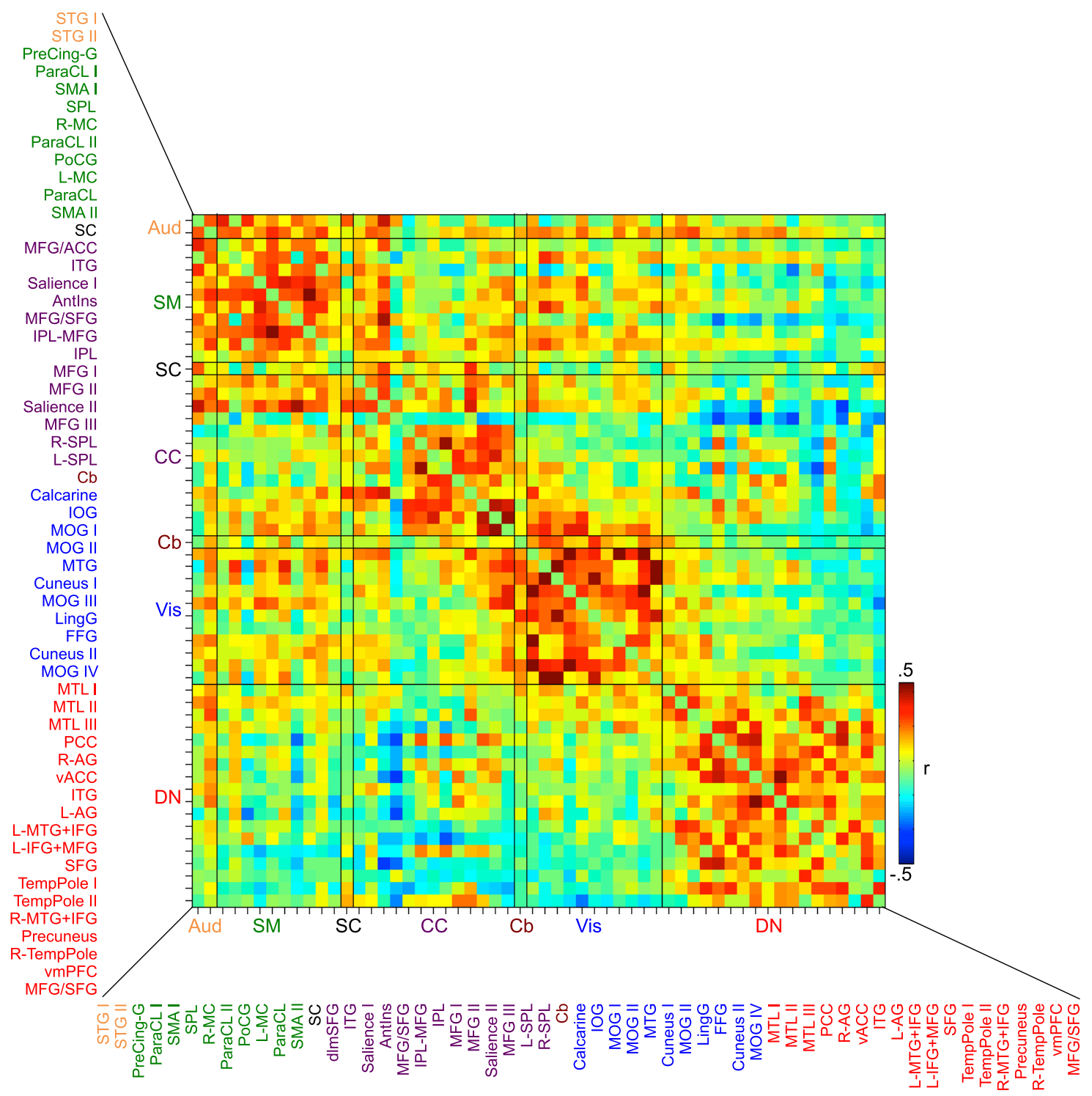

Figure 3. Static (mean) functional network connectivity correlation matrix of ICNs across task and rest. Labels are color coded to match Figure 2.

rior insula, and anterior cingulate cortex, and a second subcluster that was largely composed of lateral prefrontal and superior parietal ICNs. This was particularly evident in states F, H, E, and J. There was also striking variation in the coupling of the anterior insula to other CC networks, as such coupling ranged from being strongly and uniquely negative (states $\mathrm{K}$ and $\mathrm{B}$ ) to being more moderate in strength (state J). Finally, among DN ICNs, coupling ranged from being highly integrated (state E) to being highly disintegrated (states C, L, I), with some evidence of subclustering among predominantly posterior DN ICNs, including posterior cingulate, right and left angular gyrus, and the MTL in states F and K. All 11 states also showed marked departures from the off-diagonal coupling evident in state A or the mean connectivity pattern. Anticorrelations between CC and DN ICNs, for example, were attenuated in states F, H, E, K, and I, although more so for DN coupling with lateral prefrontal/superior parietal ICNs than coupling with dIPFC and cinguloinsular ICNs. Furthermore, coupling among SM, SC, and CB networks, which were moderately strong in state A, were markedly weaker in state C, but were stronger in states $\mathrm{D}$ and $\mathrm{E}$. Together, cluster centroids revealed marked and nonrandom departures from mean internetwork connectivity patterns.

Effects of condition and age on connectivity state expression Scatterplots showing the association of age with the frequency and MDT of each state separately for rest (blue circles) and task (red circles) are shown in Figure 5.

\section{Frequency}

Age was negatively associated with the frequency of states I, F, and $\mathrm{G}$, and positively associated with the frequency of states B and C. Condition was associated with the frequency of states $\mathrm{K}, \mathrm{B}$, and $\mathrm{C}$, with states $\mathrm{K}$ and $\mathrm{C}$ more frequent during rest than task, and state $\mathrm{B}$ more frequent during task than rest. Age and condition had an interactive effect on the frequency of states $\mathrm{H}, \mathrm{B}$, and C. For state $\mathrm{C}$, there was an age-related increase in frequency at rest, but no effect of age during task. For state B, there was an age-related increase in frequency during task, but no effect of age during rest. And for state $\mathrm{H}$, there was an age-related increase in frequency during rest, but no effect of age during task. Adding behavioral 
and sex predictors to the models revealed a positive association between RT variability and the frequency of state K at rest, and a negative association between RT variability and the frequency of state $\mathrm{E}$ during task. Error rate and sex did not explain any additional variance in the frequency of any state. With the exception of state I, where motion was positively associated with frequency, and possibly state $\mathrm{H}$, where motion was negatively associated with the frequency of expression during task, none of these associations were explainable by differences in motion between subjects.

\section{Mean dwell time}

Age was positively associated with the MDT of states $\mathrm{H}, \mathrm{D}$, and $\mathrm{C}$, and negatively associated with the MDT of state I. Condition was also associated with MDT, with longer dwell times at task than rest for states $\mathrm{L}$ and $\mathrm{B}$. There was an interaction of age and condition on the MDT of state B, with age positively associated with MDT during task but unrelated at rest. Adding behavioral and sex predictors to the models revealed a positive association between RT variability and the MDT of state I at rest, a positive association between RT variability and the MDT of state $\mathrm{H}$ during task, and a positive association of state E and error rate during task. Sex did not explain any additional variance in the MDT of any state. With the exception of state I, in which motion was positively related to the MDT, none of these associations or interactions was explainable by differences in motion.

\section{Number of transitions}

Age was positively associated with NT during task. Thus, older participants showed more state transitions during task than did younger participants (Fig. 6A).

\section{Intertransition interval}

Age was negatively associated with ITI during task. Thus, older participants showed more rapid state transitions during task than did younger participants (Fig. 6B).

\section{Variability in internetwork connectivity}

For the majority of ICN-to-ICN coupling pairs, correlation variability at rest was greater among older than younger participants (Fig. 7A). A few selected pairs, mostly among AUD, SM, and selected DN networks, showed the opposite pattern, with internetwork correlation variability greater among younger compared with older participants. There was, however, a marked reversal of this overall pattern during task. Under these conditions, a majority of ICN-to-ICN coupling pairs showed lower correlation variability among older compared with younger participants, save for several individual connections (Fig. 7B).

To bring the difference in age-related ICN-to-ICN correlation variability across rest and task into greater relief and to test the statistical significance of this effect across all 1540 unique coupling pairs, we modeled the correlation variability of each coupling pair as a weighted sum of age, condition, and the interaction of age $\times$ condition, with subject motion included as a nuisance regressor. The $\beta$-coefficient of the age $\times$ condition interaction term was expressed as a $t$ statistic, and a correction for multiple comparisons was implemented via the false discovery rate (FDR) $(q=0.01)$. The results are displayed in Figure 7C.

Coupling pairs that exhibited an age $\times$ condition interaction of their correlation variability were widely distributed, although they were clustered most densely among CC ICs, including right and left intraparietal sulcus, bilateral middle frontal gyrus, and cinguloinsular cortex, and between CC networks and SM and VIS networks. Dense clustering was also evident between DN ICs and SM and VIS networks. In summary, a widely distributed set of coupling pairs showed greater correlation variability at rest than at task for older relative to younger participants, with the most pronounced age-related increases in the dynamic range of ICNto-ICN coupling strength evidenced within and between cognitive control networks.

To examine the implications of dynamic variation in internetwork connectivity for behavior, we examined, in two separate regression models, the linear association of ICN-to-ICN variability during task on the one hand and RT variability and error rate on the other, with participant motion included as a nuisance regressor in both models. Results were corrected for multiple comparisons by means of FDR $(q=0.05)$. They appear in Figure 7, $D$ and $E$, respectively. Associations between ICN-to-ICN variability and RT variability were uniformly positive and evident in a widely distributed set of coupling pairs. Although there is no simple means of summarizing the distribution, noteworthy clusters include DN (medial temporal lobe)-CC (medial frontal gyrus, superior parietal cortex) coupling pairs, as well as DN$\mathrm{DN}$, and DN-VIS coupling pairs. Associations between ICN-toICN variability and error rate were also uniformly positive, but were more sparsely distributed. A conjunction of RT variability and error rate matrices (Fig. $7 F$ ) suggested some convergence in the association of ICN-to-ICN correlation variability and maladapted behavior (i.e., high response time variability and high error rate), most notably in DN-CC coupling pairs. 
State $\mathbf{A}$

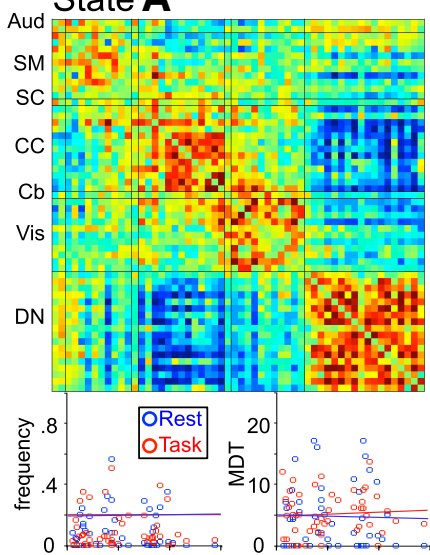

State $\mathbf{E}$
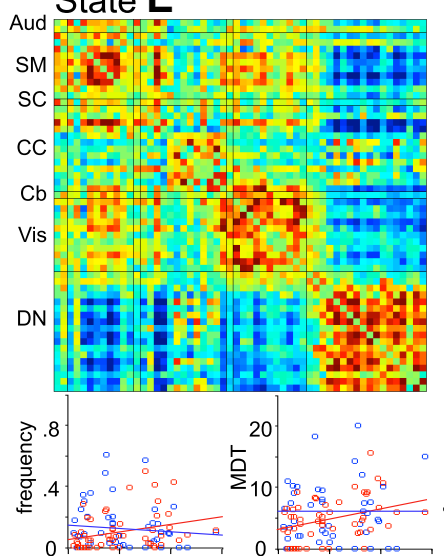

State I

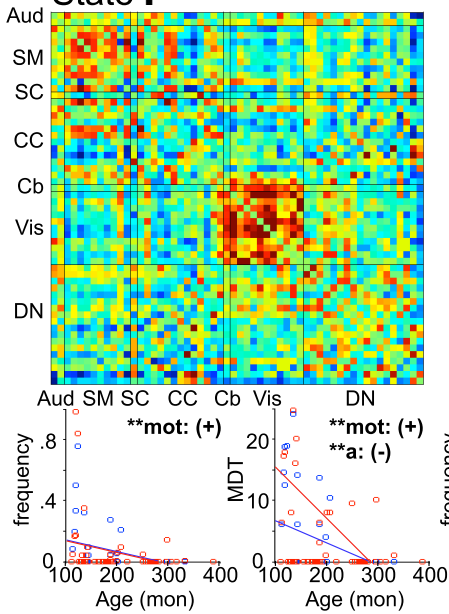

\section{State $\mathbf{B}$}
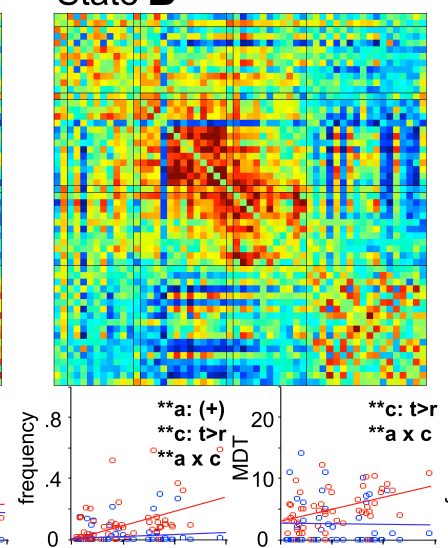

State $\mathbf{F}$
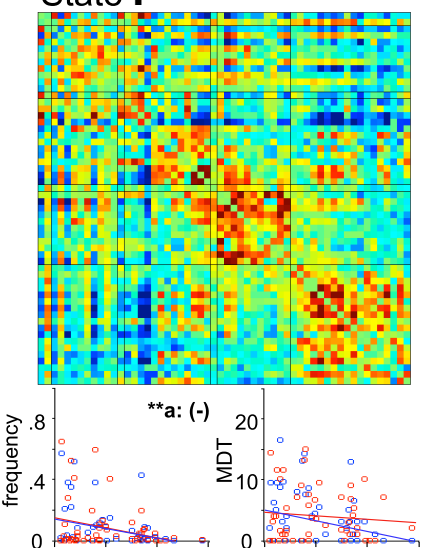

State J
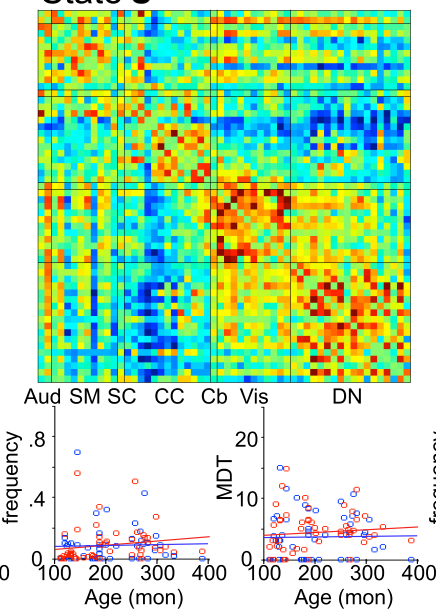

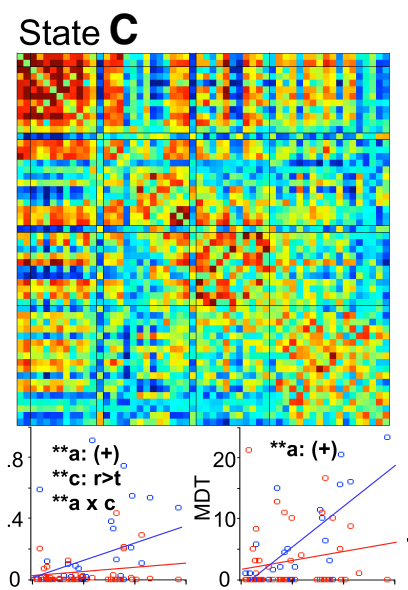

State $\mathbf{G}$
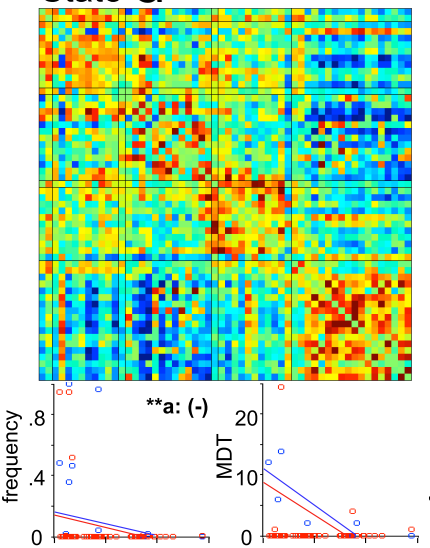

State $\mathbf{K}$
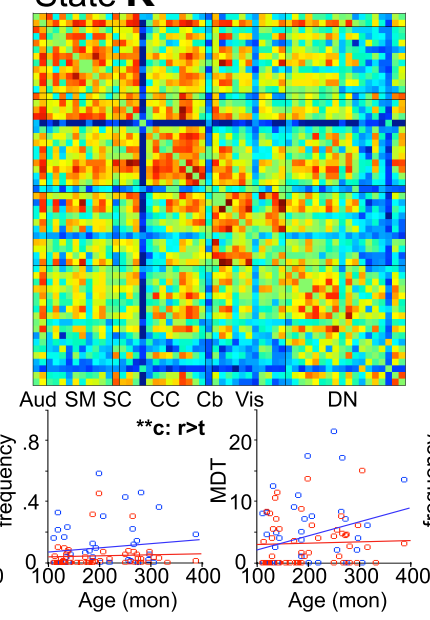

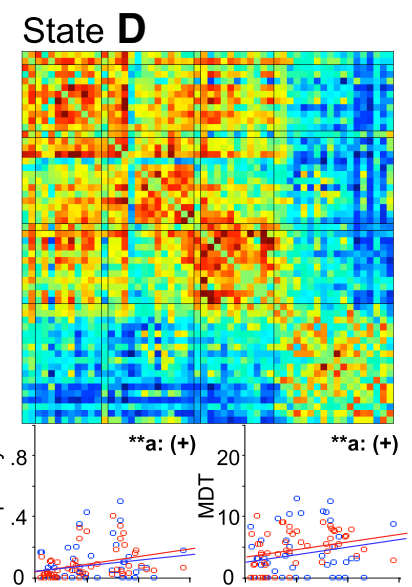

State $\mathbf{H}$

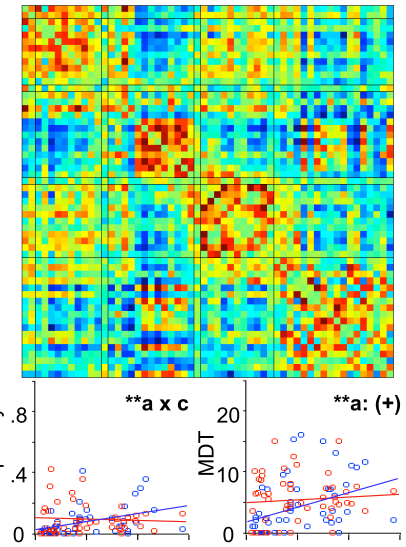

State $\mathbf{L}$
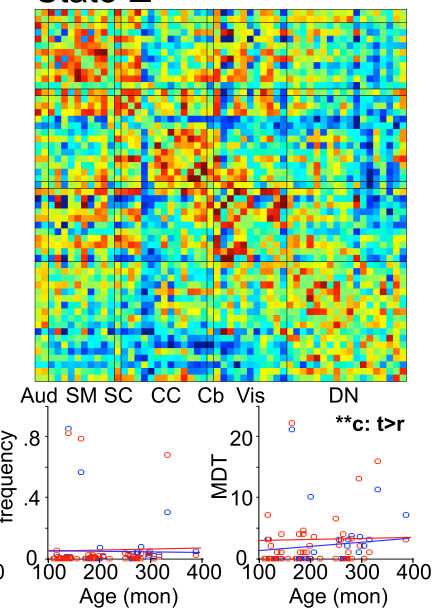

Figure 5. Age- and condition-dependent state expression. State solutions are shown for 12 states that were expressed by more than one subject. Below each ICN-to-ICN state matrix are distribution plots for frequency (left) and MDT (right) across ages for rest (blue circles) and task (red circles). Bold text marked by asterisks indicates significant main effects or interactions. a, Age; c, condition; mot, motion; r, rest; t, task.

\section{Discussion}

The present study examined the effects of age and task condition on dynamic whole-brain FC. The analysis revealed 12 recurring FC states, only one of which was similar to the mean FC. Remaining FC states departed in marked, but nonrandom ways from the mean FC pattern, with substantial internetwork correlation variability. Age-dependent changes were evident in the transitional dynamics of the states across rest and task, and in internetwork correlation variability, suggesting that changes to large-scale brain dynamics, the "dynome" (Kopell et al., 2014) or the "chronnec- tome" (Calhoun et al., 2014), may be a fundamental feature of functional brain development.

Dynamic changes in network topology are evident both locally, within the perimeter of traditionally defined network boundaries (Chang and Glover, 2010; Hutchison et al., 2013b; Kucyi and Davis, 2014), and globally, at a whole-brain level (Liu et al., 2013; Allen et al., 2014). While, in principle, it is possible that connectivity states reflect noise-driven variation around a putative mean state, aspects of the present findings argue against this view. First, several connectivity states identified here bear 
a marked resemblance to connectivity states identified previously (Allen et al., 2014), including the following: (1) a "canonical" state akin to mean FC (state A); (2) a "somatomotor disconnection" state marked by strong coupling among SM ICNs, disconnection between SM and SC ICNs, and disintegration among DN ICNs (state C); and (3) two states that, despite varied topology, showed widespread disconnection of all ICNs with DN ICNs (states D and E). Evidence that select states are reproducible across studies suggests that they constitute a core feature of the adult state repertoire, and are robust against variation in methods, subjectspecific brain morphology, and data quality. Second, principled changes in state expression occurred in association with changes in cognition and behavior. During the performance of a cognitive control task, states linked to drowsiness and inattention (state C) decreased in frequency relative to rest, whereas states marked by strong coupling among prefrontal cognitive control and visual ICNs (state B) increased in frequency relative to rest. The functional relevance of state B is particularly noteworthy, given the marked coupling among several cognitive control ICNs, including dorsolateral prefrontal, anterior cingulate, anterior insular, and superior parietal cortices. These regions have not only been linked to efficient stimulus/response selection and stable task-set maintenance in traditional studies of brain activation (Wilk and Morton, 2012; Wilk et al., 2012), but also undergo profound functional reorganization over development (Crone and Dahl, 2012). Note that strong cognitive control ICNto-ICN coupling was not exclusive to this state (see states A, D, and $\mathrm{K})$. This suggests that cognitive control is associated with a whole-brain state configuration that includes a unique connectivity fingerprint across all ICs, and not solely task-evoked activity in select "task-relevant" regions. Together then, the findings support the idea that dynamic state expression is not a reflection of random variance, but is a principled neurophysiological response to changing cognitive demands.

The findings motivate a reconceptualization of the link between cognitive control and FC. Current models assume that cognitive control networks are time-invariant structures, measureable by the mean FC assessed while participants are at rest, and whose enduring topological features somehow constrain executive processes (Cole and Schneider, 2007; Fair et al., 2007). Our results instead suggest that these networks are, like cognitive control process themselves, transient and dynamic. Furthermore, if, as emerging evidence suggests, dynamic connectivity states are nonrandom patterns (Hutchison et al., 2013a), then mean FC, on which static network characterizations are based, is a mixture of discrete connectivity states, each potentially evoked or exploited to some degree by cognition. From this perspective, the link between cognitive control and FC may be more direct than has been considered to date. Rather than indirectly correlated with static network topology, cognitive control may be directly coincident with dynamic changes in FC.

The implications for development are clear. Foremost perhaps is that alterations in the brain's spatial-temporal coupling structure that occur over the course of development (Deco et al., 2009) impact FC dynamics, with several lines of evidence consistent with this prediction. There were, for example, age-related differences in the frequency of state expression, with older participants expressing states $\mathrm{C}$ and $\mathrm{D}$ - states known to comprise the adult repertoire (Allen et al., 2014)—more frequently than younger participants, and younger participants expressing states $\mathrm{F}$ and $\mathrm{G}$ more frequently than older participants. Age-related differences were also evident in the effect of condition (rest vs task) on state expression. Older participants, for example, expressed the somatomotor disconnection state-state $\mathrm{C}$-more frequently at rest than during task, and expressed state B less frequently at rest than during task. Younger participants, by contrast, expressed states B and $\mathrm{C}$ with comparable frequency across rest and task. Condition also affected the mean dwell time of states $\mathrm{B}$ and $\mathrm{H}$ for older, but not younger participants. Importantly, age-related differences in dynamic state expression were 
A

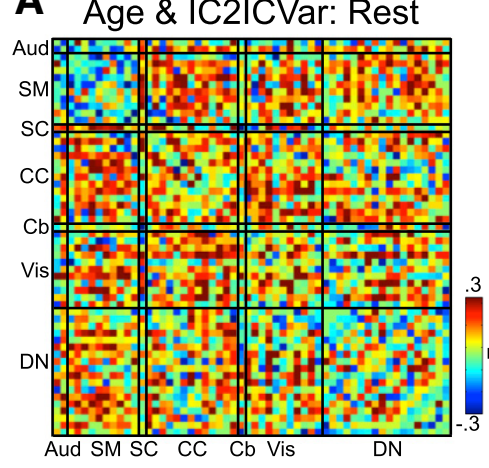

D

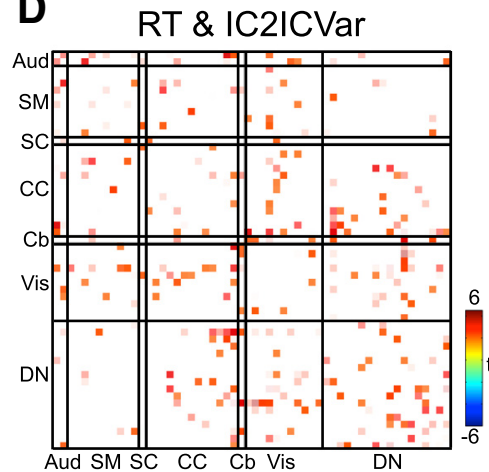

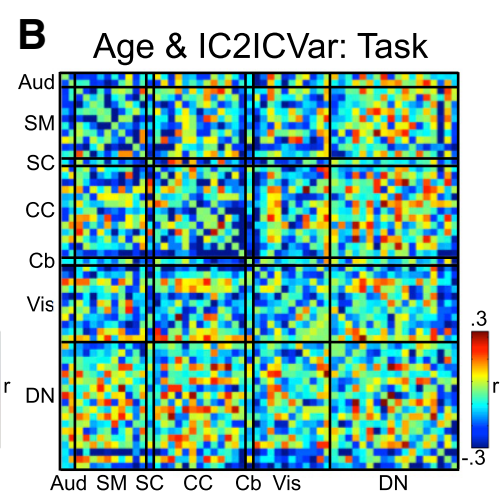

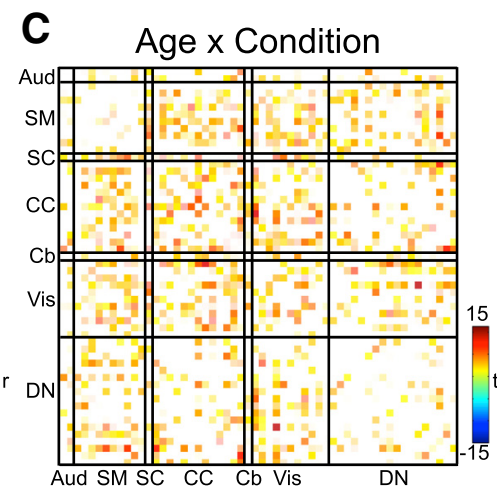

E

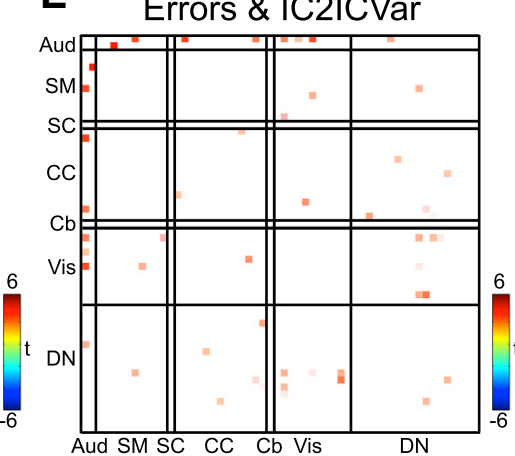

$\mathbf{F}$

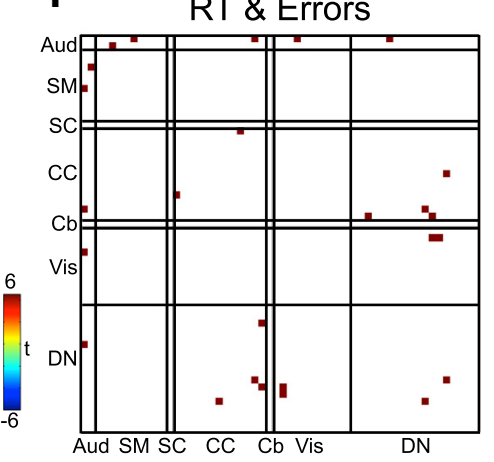

Figure 7. Age-related differences in internetwork connectivity variability. $\boldsymbol{A}, \boldsymbol{B}$, Variance in the ICN-to-ICN coupling strength was estimated for each pairwise ICN-to-ICN coupling, separately for rest $(\boldsymbol{A})$ and task $(\boldsymbol{B})$. Each coupling strength variability estimate was then correlated with age. Positive correlations (warm colors) indicate that connectivity variability was greater among older than younger participants; negative correlations (cool colors) indicate that variability was greater among younger than older participants. C, A statistical test of the interaction of age and condition, FDR corrected for multiple comparisons $(q=0.01)$, reveals coupling pairs whose correlation was more variable at rest than task for older relative to younger participants. No coupling pairs were more variable at rest than task for younger participants. $\boldsymbol{D}$, Correlation of ICN-to-ICN variability and RT variability, FDR corrected $(q=0.05)$. $\boldsymbol{E}$, Correlation of ICN-to-ICN variability and error rate, FDR corrected $(q=0.05)$. $\boldsymbol{F}$, Conjunction of $\boldsymbol{D}$ and $\boldsymbol{E}$.

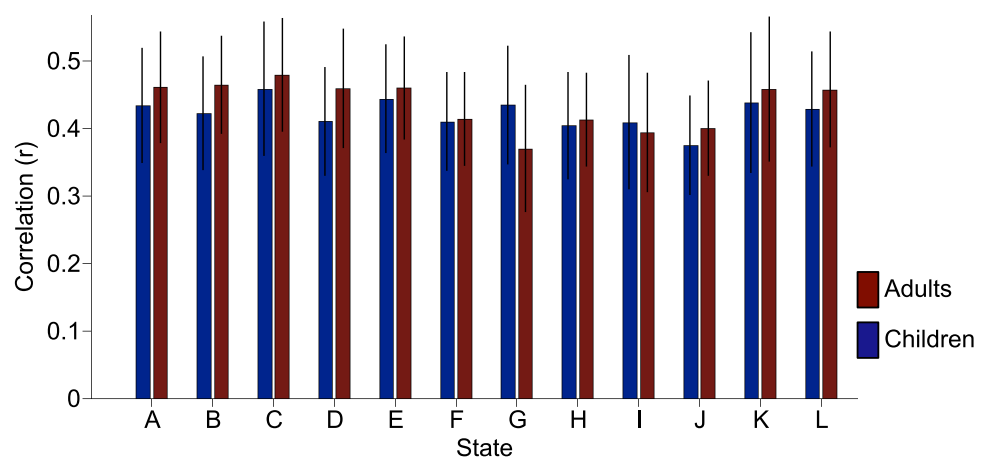

Figure 8. Average state fit of children ( $<216$ months) and adults ( $\geq 216$ months). $\boldsymbol{A}-\boldsymbol{L}$, Bars represent the mean spatial correlation of all windowed patterns with the canonical state configuration to which it was assigned by $k$-means clustering, derived separately for children (blue) and adults (red). Error bars represent 1SD.

evident even though the states themselves were topologically similar for participants of different ages (Fig. 8). Together, our findings suggest that it is dynamic state transitions rather than a static topology traced by inter-regional connections that is the locus of change in the years spanning late childhood and early adulthood.

Whether changes in state expression are a consequence of, or can even be predicted from, changes in the brain's spatial-temporal coupling structure, remains unclear. Anatomical coupling length, transmission time delays, and neurophysiological noise (Deco et al., 2009), fundamentally constrain emergent neural dynamics and undergo dramatic change during the developmental period spanned by the current sample. Examination of the relationship between changes in the brain's coupling structure and changes in state dynamics deserves close consideration in future studies.

\section{The noisy brain}

A second prediction concerned agerelated differences in inter-regional coupling variability and the complexity of evoked brain dynamics. Although major structural pathways and functional features within the human connectome develop before 2 years of age (LaMantia and Rakic, 1990; Luo and O'Leary, 2005; Low and Cheng, 2006; Gao et al., 2011; Yap et al., 2011), changes in the size (i.e., diameter and length), relative alignment, packing density, and myelination of axons continue until early adulthood, and promote the efficacy of neural communication (Innocenti, 1995; Chen et al., 2002; Bütefisch, 2004; Paus, 2010). Numerical simulations suggest that these changes, together with age-related increases in neurophysiological noise, lead to greater complexity in evoked brain dynamics. Much as an athlete is best prepared for action when in motion, noise lends computational capacity to the dynamic brain by facilitating transitions between different multistable connectivity states given external stimulation. Greater complexity in evoked dynamics, in turn, affords greater cognitive and behavioral flexibility. 
The present findings were broadly convergent with these ideas. At rest, ICN-to-ICN coupling variability was typically higher among older than younger participants, and brain dynamics were of comparable complexity. However, with the administration of a cognitive control task, there was a marked reversal of these effects. ICN-to-ICN variability was typically lower among older participants, and brain dynamics were decidedly more complex - the number of states expressed, the number of transitions occurring between multistable connectivity states, and the rate of transition between states were all higher among older than younger participants. Thus, as predicted, there was an age-related increase in the complexity of brain dynamics evoked by a challenging cognitive control task.

Variability in ICN-to-ICN coupling also had implications for behavior. During the administration of a cognitive control task, ICN-to-ICN variability among selected DN-to-DN, DN-to-CC, and VIS-to-CC couplings were positively associated with RT variability and error rate. These associations remained largely unchanged after controlling for differences in age. Associations between DN function and behavior are well documented. DN activity, for example, increases in the moments preceding error commission (Eichele et al., 2008), and is associated with momentary lapses of attention (Weissman et al., 2006). Individual differences in static DN connectivity have been associated with the tendency to engage in mental time travel (Andrews-Hanna et al., 2010). And, in close alignment with the present findings, dynamic variability in the strength of DN connectivity has been linked to the frequency of mind wandering during task performance, with greater variability associated with more frequent instances of mind wandering (Kucyi and Davis, 2014).

\section{Limitations}

A major concern with using functional imaging across development are age-associated motion artifacts (Power et al., 2012). We instituted several procedures to mitigate the effects of motion, including mock-scanner pretraining, stringent motion-related data exclusion criteria, ICA-based denoising, and the use of motion regressors in all models. Beyond motion, cardiac and respiratory signals may contribute to age-related differences as normative rates differ by age. ICA is relatively effective at removing physiological signals; however, due to aliasing and possible task-specific effects, their contribution to dynamic changes cannot be ruled out without faster sampling and/or concurrent physiological recordings.

\section{Conclusions}

Much as the mind rapidly adapts to ever-changing experiences, functional connectivity dynamically varies on relatively short time scales. The present results suggest that how the brain maximizes and constrains connectivity variability in response to cognitive and behavioral challenge changes profoundly over the course of development.

\section{References}

Allen EA, Damaraju E, Plis SM, Erhardt EB, Eichele T, Calhoun VD (2014) Tracking whole-brain connectivity dynamics in the resting state. Cereb Cortex 1991 24:663-676. CrossRef Medline

Andersen SL (2003) Trajectories of brain development: point of vulnerability or window of opportunity? Neurosci Biobehav Rev 27:3-18. CrossRef Medline

Andrews-Hanna JR, Reidler JS, Huang C, Buckner RL (2010) Evidence for the default network's role in spontaneous cognition. J Neurophysiol 104: 322-335. CrossRef Medline

Brenhouse HC, Andersen SL (2011) Developmental trajectories during ad- olescence in males and females: a cross-species understanding of underlying brain changes. Neurosci Biobehav Rev 35:1687-1703. CrossRef Medline

Bullmore E, Sporns O (2009) Complex brain networks: graph theoretical analysis of structural and functional systems. Nat Rev Neurosci 10:186198. CrossRef Medline

Bütefisch CM (2004) Plasticity in the human cerebral cortex: lessons from the normal brain and from stroke. Neuroscientist 10:163-173. CrossRef Medline

Calhoun VD, Adali T, Pearlson GD, Pekar JJ (2001) A method for making group inferences from functional MRI data using independent component analysis. Hum Brain Mapp 14:140-151. CrossRef Medline

Calhoun VD, Miller R, Pearlson G, Adalı T (2014) The Chronnectome: time-varying connectivity networks as the next frontier in fMRI data discovery. Neuron 84:262-274. CrossRef Medline

Chang C, Glover GH (2010) Time-frequency dynamics of resting-state brain connectivity measured with fMRI. Neuroimage 50:81-98. CrossRef Medline

Chen R, Cohen LG, Hallett M (2002) Nervous system reorganization following injury. Neuroscience 111:761-773. CrossRef Medline

Cole MW, Schneider W (2007) The cognitive control network: integrated cortical regions with dissociable functions. Neuroimage 37:343-360. CrossRef Medline

Crone EA, Dahl RE (2012) Understanding adolescence as a period of socialaffective engagement and goal flexibility. Nat Rev Neurosci 13:636-650. CrossRef Medline

Deco G, Jirsa V, McIntosh AR, Sporns O, Kötter R (2009) Key role of coupling, delay, and noise in resting brain fluctuations. Proc Natl Acad Sci U S A 106:10302-10307. CrossRef Medline

Diamond A (2013) Executive functions. Annu Rev Psychol 64:135-168. CrossRef Medline

Di Martino A, Fair DA, Kelly C, Satterthwaite TD, Castellanos FX, Thomason ME, Craddock RC, Luna B, Leventhal BL, Zuo XN, Milham MP (2014) Unraveling the miswired connectome: a developmental perspective. Neuron 83:1335-1353. CrossRef Medline

Eichele T, Debener S, Calhoun VD, Specht K, Engel AK, Hugdahl K, von Cramon DY, Ullsperger M (2008) Prediction of human errors by maladaptive changes in event-related brain networks. Proc Natl Acad Sci U S A 105:6173-6178. CrossRef Medline

Fair DA, Dosenbach NU, Church JA, Cohen AL, Brahmbhatt S, Miezin FM, Barch DM, Raichle ME, Petersen SE, Schlaggar BL (2007) Development of distinct control networks through segregation and integration. Proc Natl Acad Sci U S A 104:13507-13512. CrossRef Medline

Fransson P, Aden U, Blennow M, Lagercrantz H (2011) The functional architecture of the infant brain as revealed by resting-state fMRI. Cereb Cortex 1991 21:145-154. CrossRef Medline

Gao W, Gilmore JH, Giovanello KS, Smith JK, Shen D, Zhu H, Lin W (2011) Temporal and spatial evolution of brain network topology during the first two years of life. PLoS One 6:e25278. CrossRef Medline

Henik A, Tzelgov J (1982) Is three greater than five: the relation between physical and semantic size in comparison tasks. Mem Cognit 10:389-395. CrossRef Medline

Hutchison RM, Womelsdorf T, Allen EA, Bandettini PA, Calhoun VD, Corbetta M, Della Penna S, Duyn JH, Glover GH, Gonzalez-Castillo J, Handwerker DA, Keilholz S, Kiviniemi V, Leopold DA, de Pasquale F, Sporns O, Walter M, Chang C (2013a) Dynamic functional connectivity: promise, issues, and interpretations. Neuroimage 80:360-378. CrossRef Medline

Hutchison RM, Womelsdorf T, Gati JS, Everling S, Menon RS (2013b) Resting-state networks show dynamic functional connectivity in awake humans and anesthetized macaques. Hum Brain Mapp 34:2154-2177. CrossRef Medline

Innocenti GM (1995) Exuberant development of connections, and its possible permissive role in cortical evolution. Trends Neurosci 18:397-402. CrossRef Medline

Kopell NJ, Gritton HJ, Whittington MA, Kramer MA (2014) Beyond the connectome: the dynome. Neuron 83:1319-1328. CrossRef Medline

Kucyi A, Davis KD (2014) Dynamic functional connectivity of the default mode network tracks daydreaming. Neuroimage 100:471-480. CrossRef Medline

LaMantia AS, Rakic P (1990) Axon overproduction and elimination in the 
corpus callosum of the developing rhesus monkey. J Neurosci 10:21562175. Medline

Liu X, Chang C, Duyn JH (2013) Decomposition of spontaneous brain activity into distinct fMRI co-activation patterns. Front Syst Neurosci 7:101. CrossRef Medline

Low LK, Cheng HJ (2006) Axon pruning: an essential step underlying the developmental plasticity of neuronal connections. Philos Trans R Soc Lond B Biol Sci 361:1531-1544. CrossRef Medline

Luna B, Padmanabhan A, O'Hearn K (2010) What has fMRI told us about the development of cognitive control through adolescence? Brain Cogn 72:101-113. CrossRef Medline

Luo L, O'Leary DD (2005) Axon retraction and degeneration in development and disease. Annu Rev Neurosci 28:127-156. CrossRef Medline

Marsh R, Zhu H, Schultz RT, Quackenbush G, Royal J, Skudlarski P, Peterson BS (2006) A developmental fMRI study of self-regulatory control. Hum Brain Mapp 27:848-863. CrossRef Medline

McIntosh AR, Kovacevic N, Itier RJ (2008) Increased brain signal variability accompanies lower behavioral variability in development. PLoS Comput Biol 4:e1000106. CrossRef Medline

Paus T (2010) Growth of white matter in the adolescent brain: myelin or axon? Brain Cogn 72:26-35. CrossRef Medline

Power JD, Fair DA, Schlaggar BL, Petersen SE (2010) The development of human functional brain networks. Neuron 67:735-748. CrossRef Medline

Power JD, Barnes KA, Snyder AZ, Schlaggar BL, Petersen SE (2012) Spurious but systematic correlations in functional connectivity MRI networks arise from subject motion. Neuroimage 59:2142-2154. CrossRef Medline

Satterthwaite TD, Wolf DH, Loughead J, Ruparel K, Elliott MA, Hakonarson H, Gur RC, Gur RE (2012) Impact of in-scanner head motion on multiple measures of functional connectivity: relevance for studies of neurodevelopment in youth. Neuroimage 60:623-632. CrossRef Medline
Somerville LH, Casey BJ (2010) Developmental neurobiology of cognitive control and motivational systems. Curr Opin Neurobiol 20:236-241. CrossRef Medline

Supekar K, Musen M, Menon V (2009) Development of large-scale functional brain networks in children. PLoS Biol 7:e1000157. CrossRef Medline

Thomason ME, Chang CE, Glover GH, Gabrieli JD, Greicius MD, Gotlib IH (2008) Default-mode function and task-induced deactivation have overlapping brain substrates in children. Neuroimage 41:1493-1503. CrossRef Medline

Van Dijk KR, Sabuncu MR, Buckner RL (2012) The influence of head motion on intrinsic functional connectivity MRI. Neuroimage 59:431-438. CrossRef Medline

Weissman DH, Roberts KC, Visscher KM, Woldorff MG (2006) The neural bases of momentary lapses in attention. Nat Neurosci 9:971-978. CrossRef Medline

Wilk HA, Morton JB (2012) Developmental changes in patterns of brain activity associated with moment-to-moment adjustments in control. Neuroimage 63:475-484. CrossRef Medline

Wilk HA, Ezekiel F, Morton JB (2012) Brain regions associated with moment-to-moment adjustments in control and stable task-set maintenance. Neuroimage 59:1960-1967. CrossRef Medline

Yan CG, Cheung B, Kelly C, Colcombe S, Craddock RC, Di Martino A, Li Q, Zuo XN, Castellanos FX, Milham MP (2013) A comprehensive assessment of regional variation in the impact of head micromovements on functional connectomics. Neuroimage 76:183-201. CrossRef Medline

Yap PT, Fan Y, Chen Y, Gilmore JH, Lin W, Shen D (2011) Development trends of white matter connectivity in the first years of life. PLoS One 6:e24678. CrossRef Medline

Zelazo PD (2004) The development of conscious control in childhood. Trends Cogn Sci 8:12-17. CrossRef Medline 